PREPARED FOR THE U.S. DEPARTMENT OF ENERGY, UNDER CONTRACT DE-AC02-76CH03073

PPPL-3780

PPPL-3780

UC-70

Operating Regime for a Backward Raman Laser Amplifier in Preformed Plasma

by

Daniel S. Clark and Nathaniel J. Fisch

February 2003

$=$ PPPL

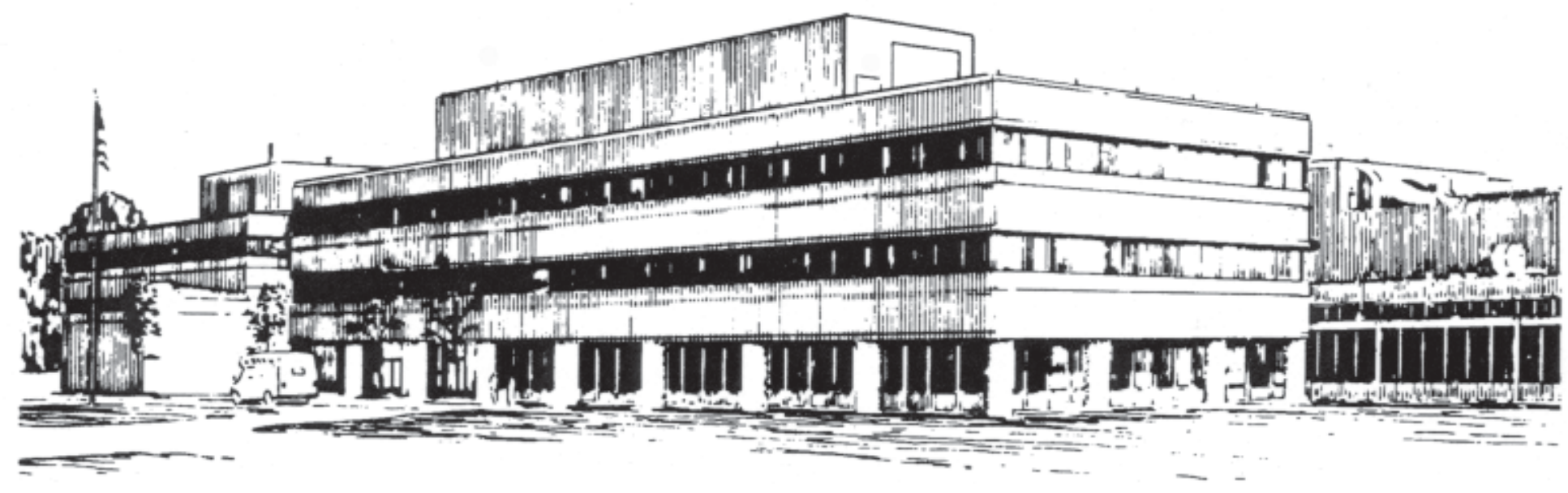

PRINCETON PLASMA PHYSICS LABORATORY PRINCETON UNIVERSITY, PRINCETON, NEW JERSEY 


\section{PPPL Reports Disclaimer}

This report was prepared as an account of work sponsored by an agency of the United States Government. Neither the United States Government nor any agency thereof, nor any of their employees, makes any warranty, express or implied, or assumes any legal liability or responsibility for the accuracy, completeness, or usefulness of any information, apparatus, product, or process disclosed, or represents that its use would not infringe privately owned rights. Reference herein to any specific commercial product, process, or service by trade name, trademark, manufacturer, or otherwise, does not necessarily constitute or imply its endorsement, recommendation, or favoring by the United States Government or any agency thereof. The views and opinions of authors expressed herein do not necessarily state or reflect those of the United States Government or any agency thereof.

\section{Availability}

This report is posted on the U.S. Department of Energy's Princeton Plasma Physics Laboratory Publications and Reports web site in Fiscal Year 2003. The home page for PPPL Reports and Publications is: http://www.pppl.gov/pub_report/

DOE and DOE Contractors can obtain copies of this report from:

U.S. Department of Energy

Office of Scientific and Technical Information

DOE Technical Information Services (DTIS)

P.O. Box 62

Oak Ridge, TN 37831

Telephone: (865) 576-8401

Fax: (865) 576-5728

Email: reports@adonis.osti.gov

This report is available to the general public from:

National Technical Information Service

U.S. Department of Commerce

5285 Port Royal Road

Springfield, VA 22161

Telephone: $1-800-553-6847$ or

(703) $605-6000$

Fax: (703) 321-8547

Internet: http://www.ntis.gov/ordering.htm 


\title{
Operating regime for a backward Raman laser amplifier in preformed plasma
}

\author{
Daniel S. Clark* and Nathaniel J. Fisch ${ }^{\dagger}$ \\ Plasma Physics Laboratory, Princeton University, \\ P.O. Box 451, Princeton, New Jersey 08543
}

(Dated: January 30, 2003)

\begin{abstract}
A critical issue in the generation of ultra-intense, ultra-short laser pulses by backward Raman scattering in plasma is the stability of the pumping pulse to premature backscatter from thermal fluctuations in the preformed plasma. Malkin et al. [V. M. Malkin, et al. , Phys. Rev. Lett., 84(6):1208-1211, 2000] demonstrated that density gradients may be used to detune the Raman resonance in such a way that backscatter of the pump from thermal noise can be stabilized while useful Raman amplification persists. Here plasma conditions for which the pump is stable to thermal Raman backscatter in a homogeneous plasma and the density gradients necessary to stabilize the pump for other plasma conditions are quantified. Other ancillary constraints on a Raman amplifier are also considered to determine a specific region in the $T_{e}-n_{e}$ plane where Raman amplification is feasible. By determining an operability region, the degree of uncertainty in density or temperature tolerable for an experimental Raman amplifier is thus also identified. The fluid code F3D, which includes the effects of thermal fluctuations, is used to verify these analytic estimates.
\end{abstract}

*Electronic address: dclark@pppl.gov

${ }^{\dagger}$ Electronic address: $f$ isch@pppl.gov 


\section{INTRODUCTION}

The recently proposed resonant backward Raman laser amplification scheme [1] utilizes the stimulated Raman backscatter in plasma of a long pumping laser pulse to amplify a short, frequency down-shifted seed pulse. In the ultra-intense regime, this scheme offers the possibility of focused intensities as high as $10^{25} \mathrm{~W} / \mathrm{cm}^{2}$ and pulse lengths of less than $100 \mathrm{fs}$ for $1 \mu \mathrm{m}$ radiation. To reach these intensities, however, this technique relies on the propagation of the pumping pulse across a preformed plasma slab of approximately $1 \mathrm{~cm}$ in length prior to its interaction with the oppositely propagating seed. During this propagation, the pump will be subject to deleterious backward Raman scattering from thermal fluctuations in the plasma prior to interacting with the seed which could degrade or completely suppress the desired amplification. Ref. [2] predicted that, due to the narrowing in time of the amplifying pulse, a nonlinear filtering effect of Raman amplification may be exploited by which a density gradient (or frequency chirp of the pump) may be introduced to detune and suppress Raman backscatter of the pump from thermal noise without suppressing the desired amplification of the seed pulse.

The problem of stability of laser pulses to nonlinear decay when propagating through plasmas is central to research in laser-driven inertial confinement fusion and has been ex-

tensively studied [3, 4]. Specifically, Mounaix et al. [5] considered the problem of the the propagation of a laser pulse of finite duration across a finite length uniform plasma subject to Brillouin backscatter from thermal noise. Successful comparison of their results with experiments were subsequently made [6]. These calculations may be readily adapted to the case of the space-time evolution of Raman backscatter, and comparisons with experiments have also been successful for such a case [7]. Likewise, the subject of the influence of plasma inhomogeneities on backscattering has also long been studied in the context of inertial confinement fusion. Particular attention has been give to regimes of absolute or convective growth and to linear saturation levels for both bounded and infinite plasmas [8].

This paper quantifies the plasma conditions as well as the steepness of any linear density gradient needed to stabilize the pump in a backward Raman amplifier. The work of Mounaix et al. is used, together with the results of Rosenbluth et al. [9] and others, to derive analytic estimates of the specific constraints placed on the plasma conditions for stable pump propagation relevant to Raman amplification. These estimates are then compared with the results 
of fluid simulations which include the effects of thermal plasma noise. Other constraints on the plasma conditions are also considered. The combination of these constraints is then visualized as a region of operability for Raman amplification in the plane of plasma density and temperature. Since the plasma conditions in any experimental realization cannot be perfectly controlled, the parameter space is surveyed to determine in what regions Raman amplification is feasible. For pump durations of $\sim 10 \mathrm{ps}$, Brillouin backscatter from thermal noise, while possible, is a secondary issue and is not considered here.

This paper is organized as follows. Sec. II describes the modeling of the space-time evolution of backscatter for a finite length homogeneous plasma and formulates these results as constraints on a Raman amplifier. Sec. III extends these results to the case of weakly inhomogeneous plasmas. Sec. IV describes several additional constraints which apply to Raman amplification. Sec. V compares the results of the analytic estimates with a series of numerical simulations, and Sec. VI summarizes and concludes.

\section{CONSTRAINTS DUE TO THERMAL NOISE IN HOMOGENEOUS PLAS- MAS}

This section addresses the limitations on plasma density and temperature in a Raman amplifier due to backscatter of the pump from thermal fluctuations in the plasma medium in the absence of density gradients. For a uniform plasma, the effect of premature backscatter of the pump form noise may be estimated using the theory developed by Mounaix et al. [5]. Specifically, Ref. [5] considers the problem of the propagation of a square laser pulse across a plasma of length $L$ in the presence of thermal fluctuations from the time of the pulse entering the plasma $(t=0)$ to times $t>L / c$. The duration of the pulse is taken to be at least the time $2 L / c$ of interest for the pump in a Raman amplifier. In the linear regime of the backscatter instability (when depletion of the pump is negligible i.e., $a_{1}=$ const.), the time evolution of the laser pulse, including a source of thermal Langmuir waves, is governed by the linearized three-wave equations

$$
\begin{aligned}
\left(\partial_{t}+c \partial_{x}\right) a_{2} & =\gamma_{R} a_{3}^{*} \\
\left(\partial_{t}-\nu_{3}\right) a_{3} & =\gamma_{R} a_{2}^{*}+S_{3} .
\end{aligned}
$$

Here $a_{1,2} \doteq|e|\left\langle A_{1,2}\right\rangle / m_{e} c^{2}$ are the normalized envelopes of the incident and scattered laser field vector potentials, $a_{3} \doteq|e|\left\langle E_{3}\right\rangle / m_{e} c \sqrt{\omega \omega_{p e}}$ is the normalized envelope of the resonant 
Langmuir wave, and $\gamma_{R} \doteq \omega a_{1}\left(n_{e} / n_{c}\right)^{1 / 4} / 2$ is the linear Raman growth rate for linearly polarized lasers. The plasma is tenuous and cold in the regime of interest $\left(n_{e} / n_{c} \ll 1\right.$ and $\left.v_{\mathrm{osc}} \doteq c a \gg v_{t e}\right)$ so that $\omega_{1} \simeq \omega_{2} \equiv \omega \gg \omega_{3} \simeq \omega_{p e}$ and advection of the Langmuir wave has been neglected for the moment, $v_{3} \simeq 0$. The damping of the light waves has also been neglected, while $\nu_{3}$ is the damping rate of the Langmuir wave (typically dominated by Landau damping). The "standard decay regime" for which this envelope formulation is valid is also assumed. Since only the threshold of instability is of interest here, the laser envelopes $a_{2,3}$ will a priori be in the linear regime and Eqs. (1) will always be valid. Finally, $n_{c} \doteq m_{e} \omega^{2} / 4 \pi|e|^{2}$ is the critical density, and $\omega_{p e}$ the electron plasma frequency.

The fluctuating Langmuir source $S_{3}$ is a stochastic function of space and time. Following Mounaix et al., for consistency with the thermal fluctuation level (in one dimension) at the resonant wave number in the absence of instability $\left(\gamma_{R} \equiv 0\right)$, the statistics of $S_{3}$ are determined by

$$
\begin{aligned}
\left\langle S_{3}(x, t)\right\rangle & =0 \\
\left\langle S_{3}(x, t) S_{3}\left(x^{\prime}, t^{\prime}\right)\right\rangle & =\Sigma_{3} \delta\left(x-x^{\prime}\right) \delta\left(t-t^{\prime}\right)
\end{aligned}
$$

where

$$
\Sigma_{3} \doteq \frac{\omega}{2 \pi} \frac{k_{3}^{2}}{n_{c}} \frac{\nu_{3}}{\omega_{p e}} \frac{T_{e}}{m_{e} c^{2}}
$$

and $\langle\cdots\rangle$ denotes ensemble average. A thermal source of backscattered light $S_{2}$ should in principle be included in Eqs. (1); however, it can be shown that

$$
\frac{\Sigma_{2}}{\Sigma_{3}}=\left(\frac{\nu_{2}}{\nu_{3}}\right)\left(\frac{\omega_{3}}{\omega_{2}}\right)\left(\frac{k_{2}}{k_{3}}\right)^{2} \simeq \frac{1}{4}\left(\frac{\omega_{p e}}{\omega}\right)^{3} \frac{\nu_{e i}}{\nu_{e i}+\nu_{L}} \ll 1
$$

for $\nu_{e i}$ and $\nu_{L}$ the collisional and Landau damping rates, respectively. The Langmuir wave source, then, clearly gives the dominant contribution to the backscatter.

The (approximate) initial and boundary conditions to be applied in solving Eqs. (1), as described by Mounaix et al., are thermal equilibrium levels of $a_{2,3}$ at the leading edge of the pump (i.e., thermal equilibrium pertains in the plasma which the pump has not yet traversed) and thermal fluctuation levels of $a_{2}$ at $x=L$. These conditions also represent stochastic functions of time with statistical properties similar to those of Eqs. (2). The additional effect of the propagation of the pump across the plasma can be modeled by an effective time dependence of the linear growth rate $\gamma_{R}=\gamma_{0} \Theta(x) \Theta(x-c t)$ with $\Theta(x)$ the Heaviside step function. 
For each realization of $S_{3}$, a Green's function method may be used to calculate the time evolution of $a_{2,3}$. Using the known statistical properties of $S_{3}$, then, the ensemble average backscatter reflectivity $\left\langle\left|a_{2} / a_{1}\right|^{2}\right\rangle$ may be calculated. In particular, for the strong damping regime $\gamma_{0} / \nu_{3} \ll 1$ applicable here, the dominant contribution to the backscatter is due to the volume source of Langmuir waves as opposed to the boundary conditions. The enhancement of the reflectivity over its thermal value is then given by

$$
\frac{1}{a_{1}^{2}}\left\langle\left|\delta a_{2}(x, t)\right|^{2}\right\rangle \simeq \frac{1}{a_{1}^{2}}\left\langle\left|\int_{0}^{L} d x^{\prime} \int_{\frac{L-x^{\prime}}{c}}^{t} d t^{\prime} g\left(x-x^{\prime}, t-t^{\prime}\right) S_{3}\left(x^{\prime}, t^{\prime}\right)\right|^{2}\right\rangle
$$

with

$$
g(x, t) \doteq \frac{\gamma_{0}}{c} \Theta(x) \Theta(c t-c) e^{-\nu_{3}(t-x / c)} I_{0}\left(\frac{2 \gamma_{0}}{c} \sqrt{x(c t-x)}\right)
$$

Using the large argument expansion of the Bessel function $I_{0}(x) \sim e^{x} / \sqrt{2 \pi x}$, Mounaix et al. derive a uniform approximation (i.e., valid for both large and small gains) for the backscatter reflectivity

$$
\frac{1}{a_{1}^{2}}\left\langle\left|\delta a_{2}(x, t)\right|^{2}\right\rangle \sim \frac{R_{\mathrm{TS}}}{G} \frac{\exp (G)-1}{\sqrt{1+\pi G}} .
$$

Here $G \doteq 2 \gamma_{0}^{2} \ell(x, t) / c \nu_{3}$ and $R_{\mathrm{TS}} \doteq \Sigma_{3} G / c$ are the gain factor and the thermal Thomson reflectivity, and $\ell(x, t) \doteq \min [x,(c t+x-L) / 2]$. Note that, for small gains $(G \ll 1)$, the reflectivity reduces to the Thomson value, while for large gains $(G \gg 1)$ it mimics the large-argument behavior of the Bessel function.

For Raman amplification, the amplification length over which the pump could be backscattered, $L$ in the above formulas, is constrained by the modulational instability of the amplifying seed [22]. In particular, for the evolving $\pi$-pulse of a Raman amplifier, the modulation growth time of the seed gives $L=c T_{\mathrm{amp}}$ with $T_{\mathrm{amp}} \sim 4 a_{1}^{-4 / 3} / \omega_{p e}$. Since, in this geometry, the pump enters the plasma from the left $(x=0)$ and remains on for a time $2 L / c$ so that the seed may continually absorb more pump energy as it propagates the entire length $L$, the greatest amount of backscatter during the amplification process will occur at $x=0$ and $t=2 T_{\mathrm{amp}}$. Using this conservative estimate of the amount of backscatter, an operating regime in the $T_{e}-n_{e}$ plane is then defined by the condition

$$
\Lambda \geq \frac{1}{a_{1}^{2}}\left\langle\left|\delta a_{2}\left(x=0, t=2 T_{\mathrm{amp}}\right)\right|^{2}\right\rangle,
$$

where $\Lambda$ represents the maximum reflectivity judged to be tolerable for a stable amplifier, e.g., $\Lambda=0.1$. 


\section{CONSTRAINTS DUE TO THERMAL NOISE IN INHOMOGENEOUS PLAS-} MAS

In the presence of a weak linear density gradient, the linearized envelope equations become $[9]$

$$
\begin{aligned}
& \left(\partial_{t}+c \partial_{x}\right) a_{2}=\gamma_{R} a_{3}^{*} \exp \left(\frac{i}{2} \kappa^{\prime} x^{2}\right) \\
& \left(\partial_{t}-\nu_{3} / 2\right) a_{3}=\gamma_{R} a_{2}^{*} \exp \left(-\frac{i}{2} \kappa^{\prime} x^{2}\right)+S_{3} .
\end{aligned}
$$

where $\kappa^{\prime} \doteq \partial \Delta k / \partial x$ is the rate of detuning from exactly resonant three-wave coupling, $0=\Delta k \doteq k_{1}-k_{2}-k_{3}$. For the case of the Raman interaction, the variation of the plasma frequency with $x$ is the dominant detuning effect so that

$$
v_{3} \kappa^{\prime} \simeq \frac{\partial \omega_{p e}}{\partial x} \equiv \frac{\omega_{p e}}{2 L_{n}}
$$

with $L_{n}$ the density scale-length and $v_{3}$ the Langmuir wave group velocity. It proves convenient [23] to quantify this detuning in terms of the small dimensionless parameter

$$
q \doteq \frac{c \omega_{p e}}{4 \gamma_{0}^{2} L_{n}} \equiv \frac{c v_{3} \kappa^{\prime}}{2 \gamma_{0}^{2}} \ll 1
$$

When assessing the lowest order the effect of spatial detuning of the Raman resonance on the stability of the pump, it is no longer valid to neglect completely the group velocity of the Langmuir wave $v_{3}$ relative to that of the light waves. Indeed, without including the lowest order contribution of $v_{3}$, the Green's function approach to calculating the backscatter reflectivity yields precisely the result found for a homogeneous plasma, i.e., no stabilizing effect is found. A simple substitution into Eq. (3) of the transformation found by Reiman [10] for converting the detuned equations (Eqs. (5)) back to the homogeneous medium equations (Eqs. (1)), shows this directly when $v_{3}=0$. Physically, this is merely the result of the equivalence of the Langmuir wave having zero group velocity and being non-dispersive $\left(v_{3}=\right.$ $\left.0 \Longleftrightarrow \partial \omega_{3} / \partial k=0\right)$. Since $\omega_{3}$ would not depend on $k_{3}$ in this case, the wave vector of the Langmuir wave may always adjust to "follow" the resonance condition as the density (and hence wave vectors of the light waves) change with the result that no net detuning occurs and no stabilization is found. Mathematically, the Green's function given in Ref. [9] for linear wave coupling in an inhomogeneous plasma may be applied, but the limit of $v_{3} \rightarrow 0$ may be taken only if simultaneously $\kappa^{\prime} \rightarrow \infty$ in such a way that $v_{3} \kappa^{\prime}$ is kept finite. The 
amplitude found for the backscatter does in fact only depend on $v_{3}$ through the combination $v_{3} \kappa^{\prime}$. Interestingly, the result used below for the backscatter Green's function (Eq. (6)) in the case of a density gradient detuning and the limit $v_{3} \rightarrow 0$ (i.e., a detuning in wavelength) is effectively identical to the Green's function found by Ref. [2] for the case of a detuning in frequency when $v_{3} \equiv 0$.

It should also be noted that, in the limit $v_{3} \rightarrow 0$, the plasma inhomogeneity is the dominating effect over the finite plasma extent. The use of the infinite medium Green's function (Eq. (6)) is then a valid approximation to this order in $v_{3}$ [11]. Further, neglecting the propagation of the Langmuir wave will remain a valid approximation only for times $t \ll 2 / v_{3} \sqrt{\kappa^{\prime}}$, i.e., so long as the Langmuir wave does not propagate out of the the region of resonance. Using $v_{3} \simeq 3 v_{t e}^{2} k_{3} / \omega_{3}$ and $v_{3} \kappa^{\prime}=2 \gamma_{0}^{2} q / c$, validity up to times $t \sim T_{\mathrm{amp}} \sim$ $4 a_{1}^{-4 / 3} / \omega_{p e}$ then requires

$$
q \ll \frac{4}{3}\left(\frac{n_{e}}{n_{c}}\right)^{-1}\left(\frac{T_{e}}{m_{e} c^{2}}\right) a_{1}^{-2 / 3}
$$

For typical parameters, this again amounts to $q \ll 1$.

As shown in Ref. [9], the Green's function for the inhomogeneous case is given by (again neglecting damping of the light wave and keeping only the combination $v_{3} \kappa^{\prime}$ )

$$
g(x, t)=\frac{\gamma_{0}}{\pi c} \sinh \left(\frac{\pi}{2 q}\right) e^{-\nu_{3}(t-x / c)+i q \eta} \int_{0}^{1} \frac{1}{z}\left(\frac{z}{1-z}\right)^{i / 2 q} e^{-2 i q \eta z}
$$

where $\eta \doteq \gamma_{0}^{2} x(c t-x) / c^{2}$ [24]. A convenient form for this solution is found by identifying Eq. (6) as an integral representation of a confluent hypergeometric function [12] [25], namely,

$$
g(x, t)=i \frac{2 \gamma_{0}}{\pi c} \sinh \left(\frac{\pi}{2 q}\right) e^{-\nu_{3}(t-x / c)+i q \eta-\pi / 2 q}{ }_{1} F_{1}\left(\frac{i}{2 q}, 1 ;-2 i q \eta\right) .
$$

For early times, $q \eta \ll 1,{ }_{1} F_{1}$ may be expanded in a series of modified Bessel functions [13]

$$
{ }_{1} F_{1}\left(\frac{i}{2 q}, 1 ;-2 i q \eta\right)=I_{0}(2 \sqrt{\eta})+i q \eta I_{2}(2 \sqrt{\eta})+\cdots
$$

showing that the evolution in the inhomogeneous case is only slightly perturbed from that of the homogeneous case. For late times, $q \eta \gg 1$, the large argument expansion of ${ }_{1} F_{1}$ yields $[14]$

$$
{ }_{1} F_{1}\left(\frac{i}{2 q}, 1 ;-2 i q \eta\right) \sim \frac{e^{\pi / 2|q|+4 q^{2} / 3}}{\sqrt{2 \pi \sqrt{1+1 / 4 q^{2}}}} e^{i \phi}
$$

indicating saturation of the instability. $\phi$ is a purely real phase factor. The condition $q \eta \sim 1$ identifies the onset of saturation for any point $x$. Since, for any point, the instability 
first grows in a form similar to the homogeneous case and then saturates at a level given by Eq. (8), an approximate representation of the Green's function is simply to take the minimum of Eqs. (7) and (8). Repeating the calculation outlined above in Eq. (3) with this approximate Green's function and neglecting any modifications to the statistical properties of $S_{3}$ due to the weak gradient leads to

$$
\frac{1}{a_{1}^{2}}\left\langle\left|a_{2}(x, t)\right|^{2}\right\rangle \sim R_{\mathrm{TS}} \min \left[\frac{\exp (\pi /|q|)}{\pi / 4|q|}, \frac{\exp (G)-1}{G \sqrt{1+\pi G}}\right] .
$$

Again, an operating regime is defined by the analogue of Eq. (4).

\section{OTHER CONSTRAINTS ON RAMAN AMPLIFICATION}

In addition to instability of the pump to thermal noise, several other factors limit the operating regime of a Raman amplifier. To calculate these constraints, the very weak density gradients considered above have been neglected, so that only the simpler uniform plasma results will be used.

Firstly, the plasma conditions must be such that the pump is not excessively absorbed due to inverse-Bremsstrahlung while propagating across the plasma. Again, choosing $\Lambda=0.1$ as a threshold for excessive absorption, the inverse-Bremsstrahlung constraint may be written

$$
\frac{1}{2} \nu_{\mathrm{IB}} T_{\mathrm{amp}} \leq-\ln \left(1-\Lambda^{1 / 2}\right)
$$

with $\nu_{\text {Ів }}$ the inverse-Bremsstrahlung absorption coefficient [15]. Note that, for simplicity this effect of damping of the pump beam has been neglected in the calculation of pump stability from Secs. II and III. This omission will be commented on in Sec. V.

Secondly, the necessity of avoiding breaking of the backscatter driven Langmuir wave and subsequent incomplete depletion of the pump imposes a lower bound on the allowable plasma electron density. Using the Manely-Rowe relations to find $a_{3}^{\max } \sim a_{1}$ and the wave-breaking criteria for a cold plasma $\omega_{p}>\omega_{b} \doteq \sqrt{|e| k_{3} E_{3} / m_{e}}$ [4] leads to

$$
n_{e} \geq n_{c}\left(4 a_{1}\right)^{4 / 3}
$$

Thirdly, excessive Landau damping of the driven Langmuir wave which would also result in incomplete depletion of the pump should be avoided. An approximate constraint is

$$
k_{3} \lambda_{\mathrm{De}} \leq \frac{1}{2}
$$


There is an added constraint to the operation of a Raman amplifier due to the possibility of forming broad nonlinear precursor pluses in place of the compressing $\pi$-pulse on which the amplification is based [16]. Since this is an effect governed by the initial shape of the seed pulse and not controlled by the ambient plasma conditions, it cannot be addressed by the formalism used here.

Taken together, for a particular incident pump intensity and laser wavelength, the constraints given above and in Secs. II and III demarcate a specific region in plasma density and temperature for which Raman amplification should be feasible (Figs. 1-4). Note that if, in a particular experimental setup, a plasma length different from the optimal scaling of $L_{\text {amp }}$ above were used, the results of Figs. 1-4 would be modified accordingly.

\section{COMPARISON WITH FLUID CODE SIMULATIONS}

The constraints presented above on the regime of operation for a backward Raman amplifier have been verified by comparison with a series of simulations using the F3D code. The details of the numerics F3D and the noise model used in the code are described at length in Ref. [17]. For each of four representative combinations of laser wavelengths and intensities $\left(\lambda=0.532 \mu \mathrm{m}\right.$ and $1.064 \mu \mathrm{m} ; I=1.0 \times 10^{14} \mathrm{~W} / \mathrm{cm}^{2}$ and $\left.2.0 \times 10^{14} \mathrm{~W} / \mathrm{cm}^{2}\right)$, a sequence of simulations was run to span the relevant portion of the electron density and temperature plane for helium plasmas. With each choice of density and temperature, the length of the simulation was adjusted to correspond to the growth length for the modulational instability of the amplifying seed and cases were run with both uniform density profiles and a series of increasing density gradients (corresponding typically to $q=0.0,0.05,0.1,0.15,0.2$, and $0.25)$.

While the primary focus here is the stability of the pump, seed pulses were also launched in the simulations to gauge the effect on net amplification by varying temperatures and densities. The seed pulses were in all cases launched exactly when the pump had completely crossed the plasma from the opposite side and were given an amplitude equal to that of the pump and a trapezoidal shape in time with a 0.2 ps flat-top and 0.021 ps "risers." Since the $\pi$-pulse is an attractor solution of the three-wave equations, provided the initial seed is sufficiently intense and sharp to reach this attractor, its actual shape should have little influence on the ultimate degree of amplification. 
To compare easily with the above analytic results and for speed of computation, all simulations were run in only one dimension. Since direct backscatter is the fastest growing of the Raman interactions, no essential modifications of the physics are expected by including more than one dimension, at least as concerns the issue of amplifier stability to pump depletion by Raman backscatter from thermal noise. The effects of inverse-Bremsstrahlung of the laser fields and Landau damping of the Langmuir wave were also included in the simulations. A model for Langmuir wave breaking is not currently available in F3D.

The comparison of the analytic estimates and the numerical results is summarized by Figs. 1-4. The labeled curves correspond to the constraints discussed above: Above the curve labeled "collisional damping," more than $10 \%$ attenuation of the pump from inverseBremsstrahlung should be expected. Below the line labeled "wave-breaking," the driven Langmuir wave can be expected to break, preventing complete pump depletion and hence degrading the amplification efficiency. The curve label " $k_{3} \lambda_{D e}=0.5$ " represents an approximate boundary beyond which Landau damping of the Langmuir wave should become strong. The curves labeled " $q=\ldots$ " are the boundaries above or to the right of which more than $10 \%$ backscatter of the pump intensity can be expected for the corresponding density gradient. That is, for $q \neq 0.0$, only points below or to the left of the corresponding curve would be stable to thermal noise. Note that the curves labeled for $q \neq 0.0$ include part of the $q=0.0$ curve before curving steeply upward in $n_{e}$. This merely reflects the minimum function appearing in Eq. (9): for sufficiently high temperatures at a given density, the development of the instability is as if the plasma were uniform, while below some threshold temperature the instability saturates at a level dependent on $q$. With increasing values of $q$, this "turning point" moves progressively further to the right (i.e., to higher temperatures) and renders a larger portion of $T_{e}-n_{e}$ plane stable to pump backscatter — as can be expected physically. The red area denotes the stable domain for $q=0.0$, i.e., a homogeneous plasma. For the corresponding $q>0.0$, this stable domain is extended to include the red and yellow areas. The simulation results are represented by the symbols $(*, \triangle, \square, \diamond)$ and were judged stable, marginal, unstable, or opaque, respectively, on the criteria of $10 \%$ backscatter of the pump from thermal noise or $10 \%$ attenuation of the pump due to inverse-Bremsstrahlung.

The results of the simulations are seen to be in generally good agreement with the analytic estimates. The points corresponding to $\left(T_{e}=20 \mathrm{eV}, n_{e}=0.02 n_{c}\right)$ in Fig. 3 and $\left(T_{e}=20 \mathrm{eV}\right.$, $\left.n_{e}=0.02 n_{c}\right)$ and $\left(T_{e}=100 \mathrm{eV}, n_{e}=0.1 n_{c}\right)$ from Fig. 4 , however, show stability or marginal 
stability when instability should be expected. This can be ascribed to the strong amounts of collisional damping experienced by the pump in these runs: since the backscatter of thermal noise is exponentially dependent on the amplitude of the pump, degradation of the pump by inverse-Bremsstrahlung can substantially reduce the growth of noise (an effect neglected in the analytic estimates) and render an otherwise stable point unstable. It should be noted that the vertical scale of Figs. 3-4 is increased from that of Figs. 1-2 since the effect of inverse-Bremsstrahlung is lessened for longer wavelengths and hence opens a larger area of parameter space for consideration. Also, the line representing $k_{3} \lambda_{D e}=0.5$ has been omitted from Fig. 4 since it is always below the wave-breaking constraint for this range of temperatures.

As an example of the effects of noise on the pump, snapshots of the evolving laser field envelopes are shown in Fig. 5 for the simulation corresponding to the point $\left(T_{e}=200 \mathrm{eV}\right.$, $\left.n_{e}=0.02 n_{c}\right)$ from Fig. 1 - just beyond the instability boundary. Initially, the pump (shown in blue) propagates stably across the plasma from the left but by $t=33 \mathrm{ps}$ is strongly depleted by thermal backscatter, while the seed (shown in green) is only beginning to propagate through the plasma. Evidently, the stability boundary marked by $q=0.0$ in Fig. 1 represents a quite steep transition between stability and instability. In Fig. 6 is shown the result of an identical run but with a slight density gradient corresponding to $q=0.15$. The thermal noise (barely visible as the red Langmuir fluctuations for $t>33 \mathrm{ps}$ ) is seen to be completely stabilized by the gradient, and the seed pulse is much more strongly amplified. Note, however, that, due to the density detuning, only $\sim 50 \%$ of the pump energy is backscattered into the seed.

Generally, for uniform plasmas, the maximum amplification of the seed (i.e., optimal operating point for a Raman amplifier) occurs for the lowest $T_{e}$ and $n_{e}$ consistent with the constraints due to wave-breaking and thermal noise and is represented by the lower left corner of the shaded area in the figures. Respectively, this corresponds simply to reducing as much as possible the Landau damping of the driven Langmuir wave and lengthening as much as possible the growth time of the modulational instability of the seed. In particular, the point corresponding to $\left(T_{e}=100 \mathrm{eV}, n_{e}=0.005 n_{c}\right)$ of Fig. 1 attained a seed amplitude 285 times that of the pump, namely $2.85 \times 10^{16} \mathrm{~W} / \mathrm{cm}^{2}$.

For non-uniform plasmas, based on the analytic estimates above, $q=0.2-0.25$ is sufficient to stabilize the pump across the entire region of the $n_{e}-T_{e}$ plane shown in Figs. 1-4. That 
is, any operating point between the boundary where inverse-Bremsstrahlung of the pump becomes excessive and $T_{e} \sim 500 \mathrm{eV}$ should be feasible for amplification. For temperatures beyond $500 \mathrm{eV}$ still larger density gradients would be needed to control thermal noise, though such points are already very far from the desirable regime for Raman amplification. From the simulations, $q=0.15-0.2$ proved sufficient to ensure stability for all of the temperature and density combinations tried, in reasonable agreement with the analytic estimates.

It is evident from the figures that a stable regime exists for Raman amplification in this idealized model even in the absence of density gradients. However, the greatest amplification was obtained for the point $\left(T_{e}=20 \mathrm{eV}, n_{e}=0.005 n_{c}\right)$ with $q=0.15$ in Fig. 1, namely a seed output intensity 390 times greater than the pump corresponding to $3.9 \times 10^{16} \mathrm{~W} / \mathrm{cm}^{2}$. This is a reflection of the fact that stability to thermal noise is governed most strongly by Landau damping of the Langmuir wave, i.e., the rate of growth of a given fluctuation is exponentially dependent on the Landau damping rate of the driven Langmuir wave. However, the useful Raman amplification is also dependent on the growth of the driven Langmuir wave, such that entering a regime of density and temperature where Landau damping is minimal but where backscatter from thermal noise is controlled by a density gradient should be optimal. Clearly, too steep a density gradient will completely suppress the Raman resonance such that a balance must then be sought between minimizing Landau damping and not requiring an excessively strong gradient.

Note that the boundary for instability to thermal backscatter is to a good approximation merely a linear relationship between $n_{e} / n_{c}$ and $T_{e}$. Again, this represents the importance of Landau damping in determining the stability to noise: for the case of Fig. 1, the boundary is roughly coincident with the line $k_{e} \lambda_{D e} \simeq 1 / 2 \sqrt{3}$. A simplification of Eq. (4) to represent this simple fact (namely, that the delineating value should be $1 / 2 \sqrt{3}$ as opposed to some other value), however, could not be found, and illustrates the importance of the initial thermal fluctuation levels in determining the ultimate stability of the pump.

\section{CONCLUSIONS}

Many simplifications have been made in calculating the above constraints. While the approximation of a one-dimensional interaction should capture the dominant effects of direct Raman backscatter in determining the stability of the pump, two- or three-dimensional 
effects related to finite focusing widths and lengths and the proper overlap of pump and seed pulse may substantially degrade the efficiency of interaction from the simulation results presented above. The assumption of perfectly tailored density profiles (either perfectly square for the homogeneous case or with a precisely prescribed density gradient for the inhomogeneous case) is also unrealistic. Indeed, one of the greatest challenges in demonstrating Raman amplification experimentally may be in the production of adequately uniform plasmas. Finally, based on the results found is Sec. V, the interaction of attenuation of the pump by inverse-Bremsstrahlung and pump stability to thermal noise may not always be negligible.

In summary, the constraints on plasma parameters for the stability of the pump in a plasma-based backward Raman laser amplifier, as well as other ancillary constraints, have been quantified. The combination of these constraints has been visualized as a region in the

plane of plasma density and temperature from which the most favorable regime for Raman amplification can be easily identified. Specific choices of wavelength and pump intensity have been used for illustration of these constraints, and agreement between the analytic estimates and numerical simulations has been demonstrated. It is evident that Landau damping of the driven Langmuir wave in the Raman interaction plays a crucial role in determining the stability of the pump. In particular, for $\lambda=0.532 \mu \mathrm{m}$ and $I=1.0 \times 10^{14} \mathrm{~W} / \mathrm{cm}^{2}$, plasma densities and temperatures such that $k_{e} \lambda_{D e} \geq 1 / 2 \sqrt{3}$ approximately determine a region of pump stability. Within this constraint, the lowest densities and temperatures consistent with avoiding the breaking of the driven Langmuir wave $\left(n_{e} \geq n_{c}\left(4 a_{1}\right)^{4 / 3}\right)$ provide optimal amplification since damping of the driven Langmuir wave and limitation by the modulational instability of the amplifying seed are then minimized. Outside of this constraint, density gradients corresponding to $q \simeq 0.25$ were found sufficient to stabilize the pump up to temperatures of $T_{e} \sim 500 \mathrm{eV}$. The greatest amplification factor of 390 times in the seed intensity over the input pump intensity (corresponding to $I_{\text {out }}=3.9 \times 10^{16} \mathrm{~W} / \mathrm{cm}^{2}$ ) was observed for the case of a simulation with $\lambda=0.532 \mu \mathrm{m}, T_{e}=20 \mathrm{eV}, n_{e}=0.005 n_{c}$, and $q=0.15$.

\section{ACKNOWLEDGMENTS}

The help of R. L. Berger in preparing simulations with F3D and on the general theory and modeling of backscatter from thermal noise is gratefully acknowledged. This work was 
supported by the U.S. Department of Energy Contract No. DE-AC02-76-CHO-3073 and the Defense Advanced Research Projects Agency (DARPA). 
[1] V. M. Malkin, G. Shvets, and N. J. Fisch, Phys. Rev. Lett. 82(22), 4448 (1999).

[2] V. M. Malkin, G. Shvets, and N. J. Fisch, Phys. Rev. Lett. 84(6), 1208 (2000).

[3] J. D. Lindl, Inertial Confinement Fusion: the Quest for Ignition and Energy Gain Using Indirect Drive (American Institute of Physics, New York, 1998).

[4] W. L. Kruer, The Physics of Laser Plasma Interactions (Addison-Wesley, New York, 1988).

[5] P. Mounaix, D. Pesme, W. Rozmus, and M. Casanova, Phys. Fluids 5(9), 3304 (1993).

[6] H. A. Baldis, D. M. Villeneuve, B. L. Fontaine, et al., Phys. Fluids B 5(9), 3319 (1993).

[7] C. Rousseaux, G. Malka, J. L. Miquel, et al., Phys. Rev. Lett. 74(23), 4655 (1995).

[8] K. Nishikawa and C. S. Liu, in Advances in Plasma Physcis (John Wiley \& Sons, 1976), vol. 6, p. 3.

[9] M. N. Rosenbluth, R. B. White, and C. S. Liu, Phys. Rev. Lett. 31(19), 1190 (1973).

[10] A. Reiman, Rev. Mod. Phys 51(2), 311 (1979).

[11] D. Pesme, G. Laval, and R. Pellat, Phys. Rev. Lett. (4), 203 (1973).

[12] J. Mathews and R. L. Walker, Mathematical Methods of Physics (Addison-Wesley, New York, $1970)$.

[13] M. Abramowitz and I. A. Stegun, Handbook of Mathematical Functions (Wiley, New York, 1984).

[14] N. N. Lebedev, Special Functions and their Applications (Dover, New York, 1972).

[15] J. D. Huba, ed., NRL Plasma Formulary (Naval Research Laboratory Publication NRL/PU/6790-00-426 (U. S. GPO), Washington, D. C., 2000).

[16] Y. A. Tsidulko, V. M. Malkin, and N. J. Fisch, Phys. Rev. Lett. 88(23), 235004 (2002).

[17] R. L. Berger, C. H. Still, E. A. Williams, and A. B. Langdon, Phys. Plasmas 5(12), 4337 (1998).

[18] V. M. Malkin, Y. A. Tsidulko, and N. J. Fisch, Phys. Rev. Lett. 85(19), 4068 (2000).

[19] A. Bers, in Handbook of Plasma Physics, edited by M. N. Rosenbluth and R. Z. Sagdeev (North-Holland, New York, 1983), vol. 1, p. 451.

[20] F. W. Chambers, Ph.D. thesis, MIT (1975).

[21] R. Lindberg and J. S. Wurtele, Private Communication (2002).

[22] As noted in Ref. [1], forward Raman scattering (FRS) of the amplified seed may also limit the 
amplification length. Since the growth times of FRS and modulational instabilities are roughly comparable in the regime of interest and since FRS may be stabilized by the introduction of a weak density gradient [18] (as considered in Sec. III), for simplicity the amplification length is taken here to be constrained only by the modulational instability. Generalization to include the constraint of FRS, however, would be straight-forward.

[23] Chirping of the pump frequency would have an effect similar to that of a density gradient on the resonance. By properly defining $q$, the following results could also be applied to this case $[2]$.

[24] Refs. $[19,20]$ have pointed out that there are many misprints/errors in Ref. [9]. Though the essential results are correct, the supporting formulas must be used cautiously.

[25] This result was first noticed by seeking a solution of Eqs. (5) explicitly in terms of $\eta[21]$. 
Fig. 1 Comparison of analytic estimates of constraints for stable Raman amplification with results of F3D simulations for $\lambda=0.532 \mu \mathrm{m}$ and $I=1.0 \times 10^{14} \mathrm{~W} / \mathrm{cm}^{2}$ and for $q=0.0$ and $q=0.27$.

Fig. 2 Comparison of analytic estimates of constraints for stable Raman amplification with results of F3D simulations for $\lambda=0.532 \mu \mathrm{m}$ and $I=2.0 \times 10^{14} \mathrm{~W} / \mathrm{cm}^{2}$ and for $q=0.0$ and $q=0.26$.

Fig. 3 Comparison of analytic estimates of constraints for stable Raman amplification with results of F3D simulations for $\lambda=1.064 \mu \mathrm{m}$ and $I=1.0 \times 10^{14} \mathrm{~W} / \mathrm{cm}^{2}$ and for $q=0.0$ and $q=0.24$.

Fig. 4 Comparison of analytic estimates of constraints for stable Raman amplification with results of F3D simulations for $\lambda=1.064 \mu \mathrm{m}$ and $I=2.0 \times 10^{14} \mathrm{~W} / \mathrm{cm}^{2}$ and for $q=0.0$ and $q=0.23$.

Fig. 5 Snapshots of laser field envelopes illustrating the effect of premature pump backscatter from thermal noise on Raman amplification when $\lambda=0.532 \mu \mathrm{m}, I=1.0 \times$ $10^{14} \mathrm{~W} / \mathrm{cm}^{2}, T_{e}=200 \mathrm{eV}, n_{e}=0.02 n_{c}$, and $q=0.0$.

Fig. 6 Snapshots of laser field envelopes for the same parameters as Fig. 5 but with $q=0.15$. 
FIG. 1:

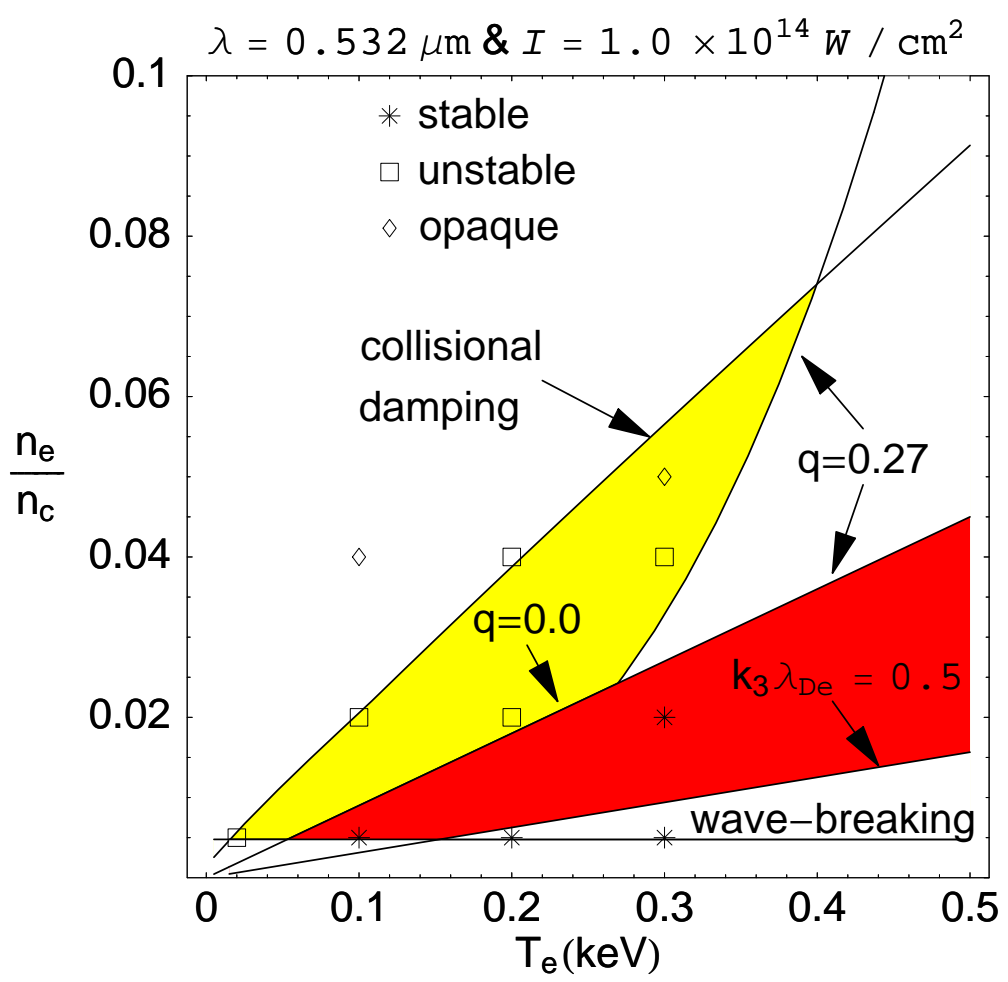


FIG. 2:

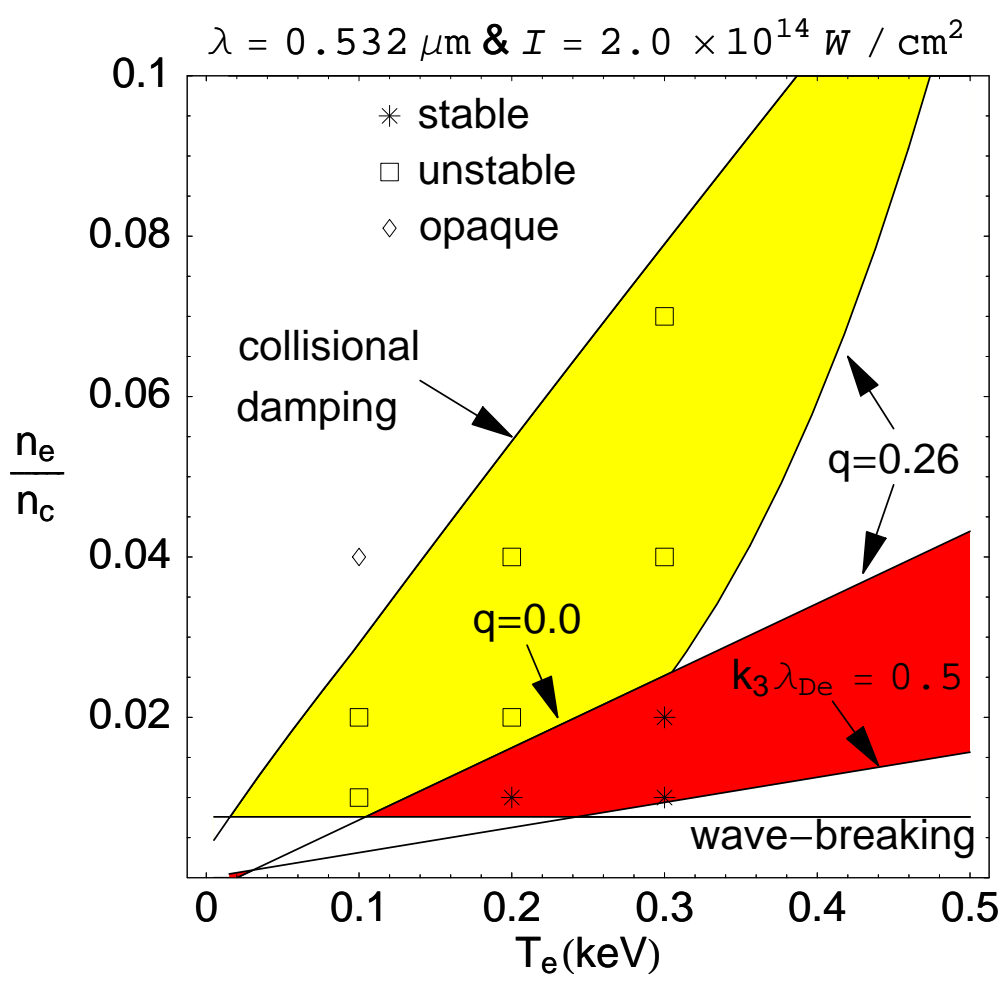


FIG. 3:

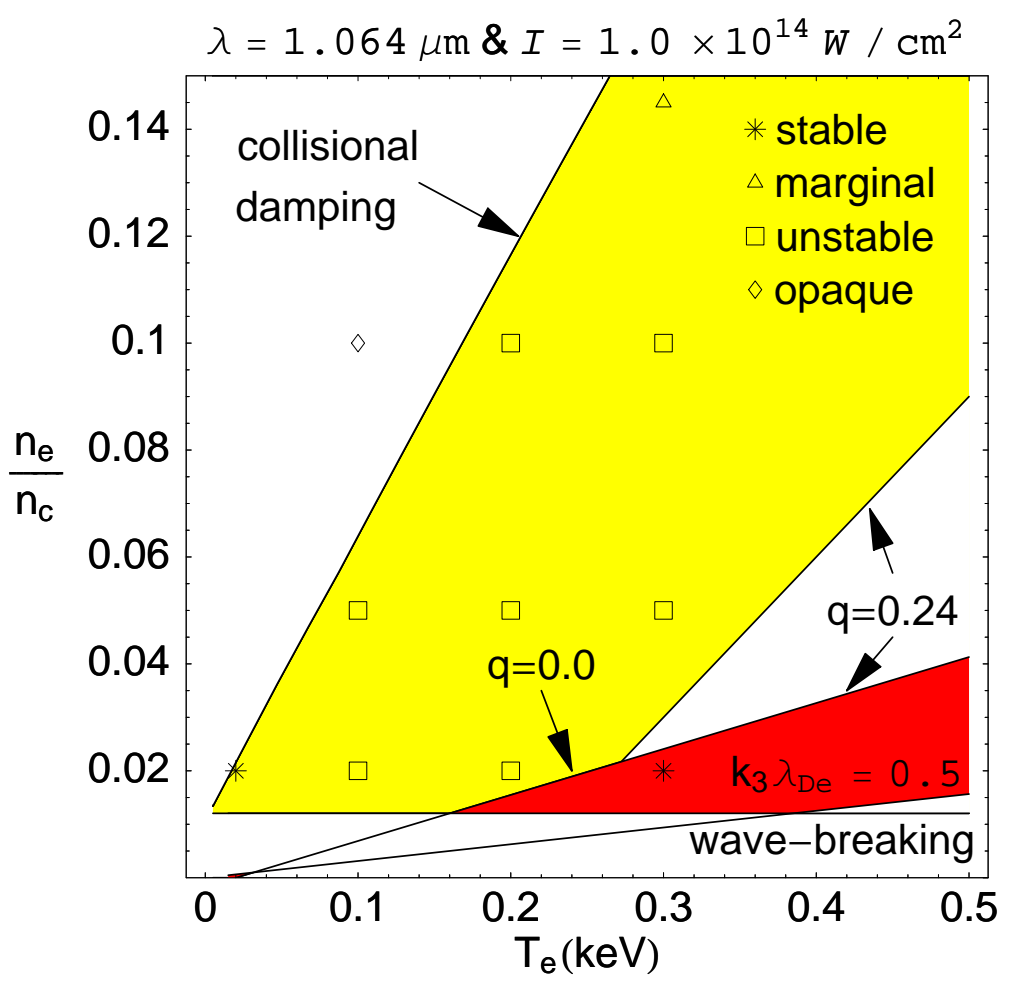


FIG. 4:

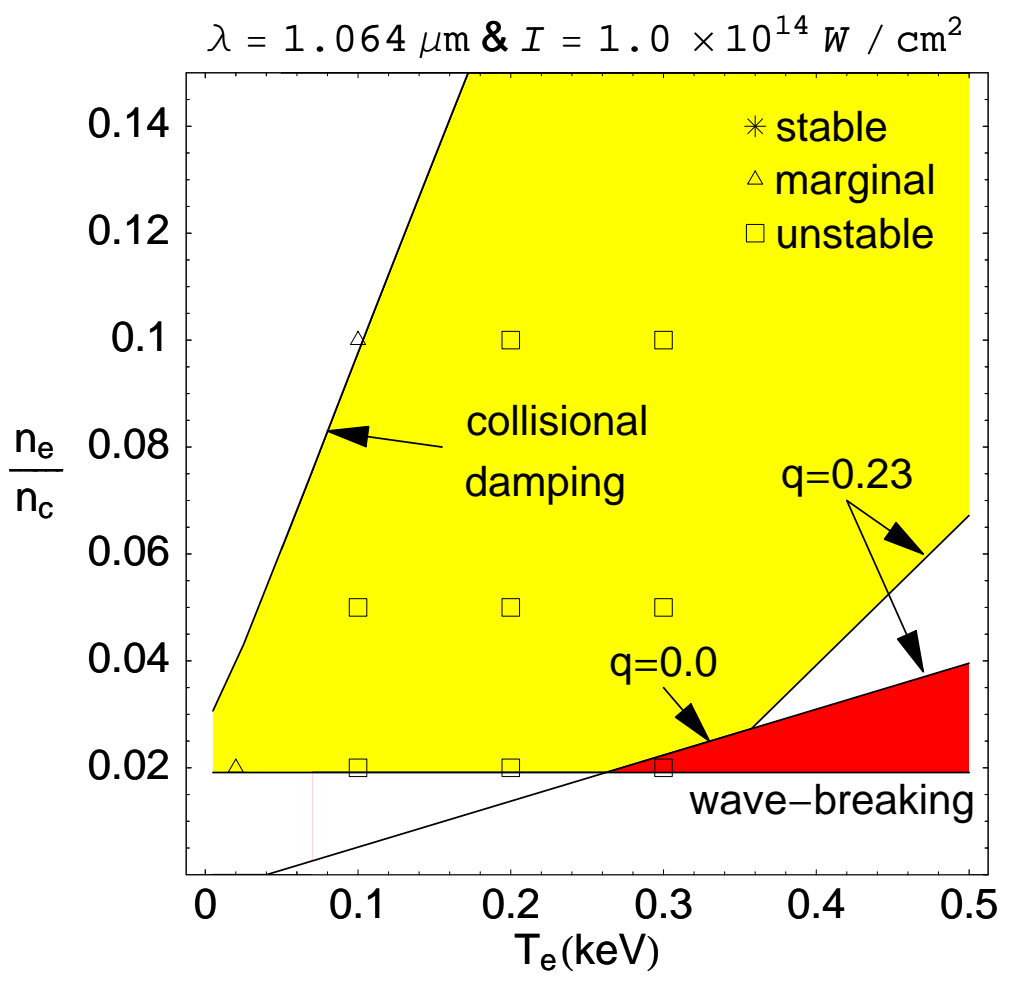


FIG. 5:

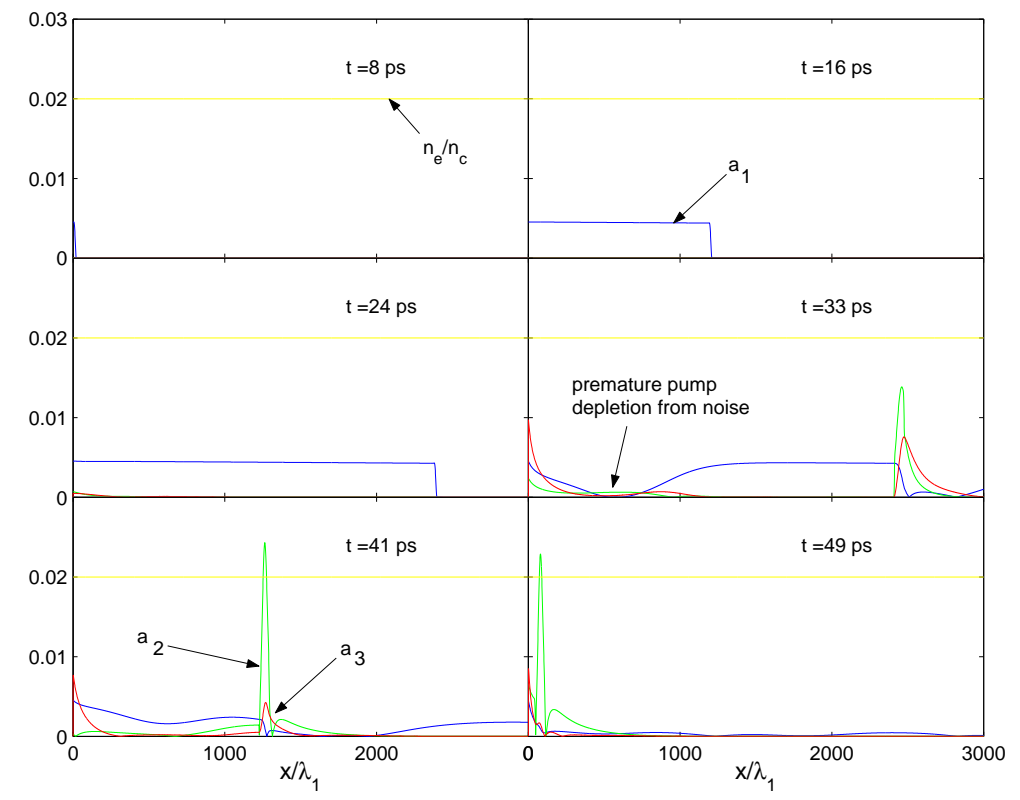


FIG. 6:

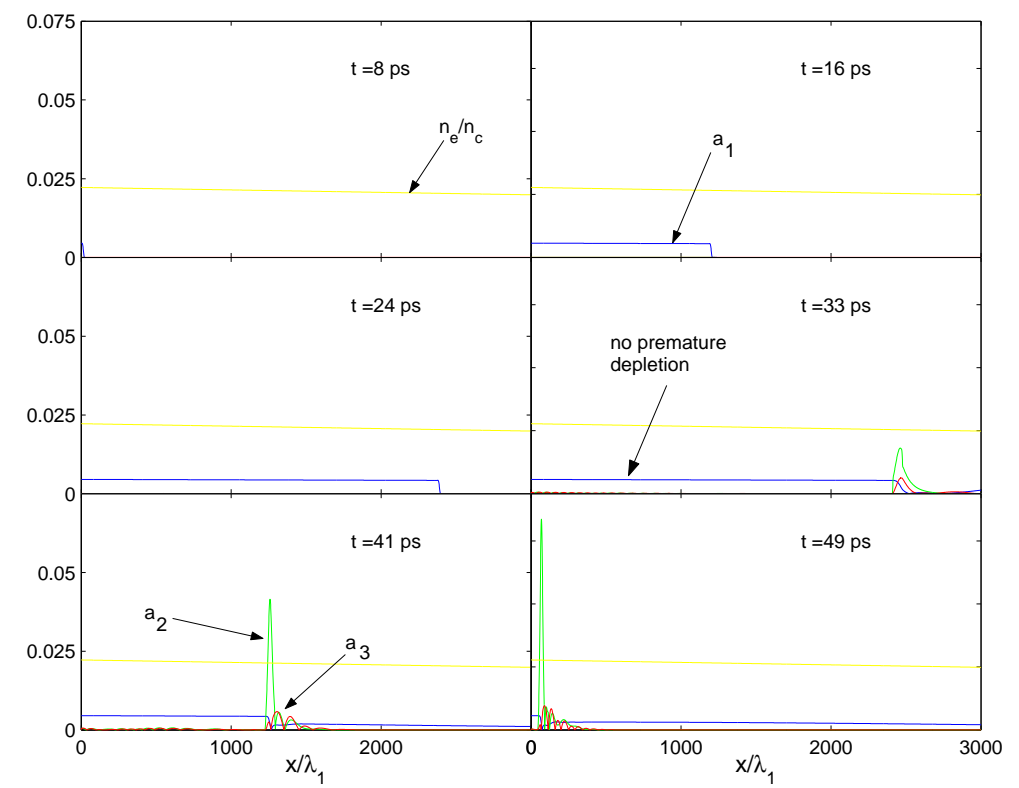




\section{External Distribution}

Plasma Research Laboratory, Australian National University, Australia

Professor I.R. Jones, Flinders University, Australia

Professor João Canalle, Instituto de Fisica DEQ/IF - UERJ, Brazil

Mr. Gerson O. Ludwig, Instituto Nacional de Pesquisas, Brazil

Dr. P.H. Sakanaka, Instituto Fisica, Brazil

The Librarian, Culham Laboratory, England

Mrs. S.A. Hutchinson, JET Library, England

Professor M.N. Bussac, Ecole Polytechnique, France

Librarian, Max-Planck-Institut für Plasmaphysik, Germany

Jolan Moldvai, Reports Library, MTA KFKI-ATKI, Hungary

Dr. P. Kaw, Institute for Plasma Research, India

Ms. P.J. Pathak, Librarian, Insitute for Plasma Research, India

Ms. Clelia De Palo, Associazione EURATOM-ENEA, Italy

Dr. G. Grosso, Instituto di Fisica del Plasma, Italy

Librarian, Naka Fusion Research Establishment, JAERI, Japan

Library, Plasma Physics Laboratory, Kyoto University, Japan

Research Information Center, National Institute for Fusion Science, Japan

Dr. O. Mitarai, Kyushu Tokai University, Japan

Library, Academia Sinica, Institute of Plasma Physics, People's Republic of China

Shih-Tung Tsai, Institute of Physics, Chinese Academy of Sciences, People's Republic of China

Dr. S. Mirnov, TRINITI, Troitsk, Russian Federation, Russia

Dr. V.S. Strelkov, Kurchatov Institute, Russian Federation, Russia

Professor Peter Lukac, Katedra Fyziky Plazmy MFF UK, Mlynska dolina F-2, Komenskeho Univerzita, SK-842 15 Bratislava, Slovakia

Dr. G.S. Lee, Korea Basic Science Institute, South Korea

Institute for Plasma Research, University of Maryland, USA

Librarian, Fusion Energy Division, Oak Ridge National Laboratory, USA

Librarian, Institute of Fusion Studies, University of Texas, USA

Librarian, Magnetic Fusion Program, Lawrence Livermore National Laboratory, USA

Library, General Atomics, USA

Plasma Physics Group, Fusion Energy Research Program, University of California at San Diego, USA

Plasma Physics Library, Columbia University, USA

Alkesh Punjabi, Center for Fusion Research and Training, Hampton University, USA

Dr. W.M. Stacey, Fusion Research Center, Georgia Institute of Technology, USA

Dr. John Willis, U.S. Department of Energy, Office of Fusion Energy Sciences, USA

Mr. Paul H. Wright, Indianapolis, Indiana, USA 
The Princeton Plasma Physics Laboratory is operated by Princeton University under contract with the U.S. Department of Energy.

\author{
Information Services \\ Princeton Plasma Physics Laboratory \\ P.O. Box 451 \\ Princeton, NJ 08543
}

Phone: 609-243-2750

Fax: 609-243-2751

e-mail: pppl_info@pppl.gov

Internet Address: http://www.pppl.gov 